\title{
A Promising Therapeutic Approach for Multiple Sclerosis: Recombinant T-Cell Receptor Ligands Modulate Experimental Autoimmune Encephalomyelitis by Reducing Interleukin-17 Production and Inhibiting Migration of Encephalitogenic Cells into the CNS
}

\author{
Sushmita Sinha, ${ }^{1,2}$ Sandhya Subramanian, ${ }^{1}$ Thomas M. Proctor, ${ }^{1,7}$ Laurie J. Kaler, ${ }^{1}$ Marjorie Grafe, ${ }^{3,4}$ Rony Dahan, ${ }^{2,3}$ \\ Jianya Huan,,$^{2,7}$ Arthur A. Vandenbark, ${ }^{1,2,5,7}$ Gregory G. Burrows, ${ }^{2,6,7}$ and Halina Offner ${ }^{1,2,4,7}$ \\ ${ }^{1}$ Neuroimmunology Research, Veterans Affairs Medical Center, Portland, Oregon 97239, and Departments of ${ }^{2}$ Neurology, ${ }^{3}$ Pathology, ${ }^{4}$ Anesthesiology and \\ Perioperative Medicine, ${ }^{5}$ Molecular Microbiology and Immunology, and ${ }^{6}$ Biochemistry and Molecular Biology and ${ }^{7}$ Tykeson MS Research Laboratory, \\ Oregon Health \& Science University, Portland, Oregon 97239
}

\begin{abstract}
Recombinant T-cell receptor ligands (RTLs) can prevent and reverse clinical and histological signs of experimental autoimmune encephalomyelitis (EAE) in an antigen-specific manner and are currently in clinical trials for treatment of subjects with multiple sclerosis (MS). To evaluate regulatory mechanisms, we designed and tested RTL551, containing the $\alpha 1$ and $\beta 1$ domains of the I-A ${ }^{\mathrm{b}}$ class II molecule covalently linked to the encephalitogenic MOG-35-55 peptide in C57BL/6 mice. Treatment of active or passive EAE with RTL551 after disease onset significantly reduced clinical signs and spinal cord lesions. Moreover, RTL551 treatment strongly and selectively reduced secretion of interleukin-17 and tumor necrosis factor $\alpha$ by transferred green fluorescent protein-positive (GFP + ) MOG-35-55-reactive T-cells and almost completely abrogated existent GFP + cellular infiltrates in affected spinal cord sections. Reduced inflammation in spinal cords of RTL551-treated mice was accompanied by a highly significant downregulation of chemokines and their receptors and inhibition of VCAM-1 (vascular cell adhesion molecule-1) and ICAM-1 (intercellular adhesion molecule-1) expression by endothelial cells. Thus, RTL therapy cannot only inhibit systemic production of encephalitogenic cytokines by the targeted myelin oligodendrocyte glycoprotein-reactive T-cells but also impedes downstream local recruitment and retention of inflammatory cells in the CNS. These findings indicate that targeted immunotherapy of antigen-specific T-cells can result in a reversal of CNS lesion formation and lend strong support to the application of the RTL approach for therapy in MS.
\end{abstract}

Key words: EAE; RTL; CNS; IL-17; chemokines/receptors; SH

\section{Introduction}

Recombinant T-cell receptor ligands (RTLs) containing the membrane distal $\alpha 1$ plus $\beta 1$ domains of class II major histocompatibility complex (MHC) molecules linked covalently to specific peptides can be used to regulate $\mathrm{T}$-cell responses and inhibit clin-

Received Aug. 8, 2007; revised Sept. 19, 2007; accepted 0ct. 2, 2007.

This work was supported by National Multiple Sclerosis Society Grants RG3794A and RG3468; National Institutes of Health Grants NS47661, Al43960, NS41965, and NS46877; The Nancy Davis MS Center Without Walls; and the Biomedical Laboratory R\&D Service, Department of Veterans Affairs. H.O., G.G.B., A.A.V., and Oregon Health \& Science University (OHSU) have a significant financial interest in Artielle ImmunoTherapeutics, Inc., a company that may have a commercial interest in the result of this research and technology. This potential conflict of interest has been reviewed and managed by the OHSU and Veterans Affairs Medical Center Conflict of Interest in Research Committees. We thank Aurelie Synder for help with confocal microscopy and Eva Niehaus for assistance in preparing this manuscript.

Correspondence should be addressed to Dr. Halina Offner, Neuroimmunology Research R\&D-31, Portland Veterans Affairs Medical Center, 3710 Southwest U.S. Veterans Hospital Road, Portland, OR 97239. E-mail: offnerva@ohsu.edu.

R. Dahan's present address: Faculty of Biology, Technion-Israel Institute of Technology, Haifa, Israel. DOI:10.1523/JNEUROSCI.3599-07.2007

Copyright $\odot 2007$ Society for Neuroscience $\quad 0270-6474 / 07 / 2712531-09 \$ 15.00 / 0$ ical experimental autoimmune encephalomyelitis (EAE). RTLs were shown to signal directly through the T-cell receptor (TCR) as a partial agonist and could prevent and treat active or passive myelin basic protein (MBP)-induced monophasic EAE in Lewis rats (Burrows et al., 1998; Wang et al., 2003), myelin oligodendrocyte glycoprotein (MOG)-induced chronic EAE in DR2 transgenic mice (Vandenbark et al., 2003), and proteolipid protein (PLP)-induced relapsing EAE in SJL/J mice. RTL constructs derived from DR2 (Haines et al., 1996) inhibited activation but induced interleukin-10 (IL-10) secretion in human DR2restricted T-cell clones specific for MBP-85-99 or cABL (BCRABL b3a2) peptides (Burrows et al., 2001; Chang et al., 2001). One such DR2 construct, RTL1000, containing the MOG-35-55 peptide, is currently in a Phase I safety trial in multiple sclerosis (MS) subjects.

Understanding the mechanism of action of RTLs is necessary for exploiting their full therapeutic potential in MS. Thus, we constructed a new I-A ${ }^{\mathrm{b}}$-derived RTL551 containing covalently 
tethered MOG-35-55 peptide and studied its role in MOG-3555 -induced EAE in C57BL/6 mice. Development of EAE not only requires generation of autoreactive $\mathrm{T}$-cells, but also involves breakdown of blood-brain barrier integrity to facilitate infiltration of T-cells and mononuclear cells into the CNS. The local cytokine and chemokine milieu in the CNS along with the matrix metalloproteinases cause damage to the blood-brain barrier integrity leading to the trafficking of inflammatory cells in the CNS and establishment of chronic EAE. Previously, we demonstrated that RTL therapy in SJL/J mice resulted in a cytokine switch involving IL-10, IL-13, and TGF $\beta 3$ (Huan et al., 2004; Offner et al., 2005). However, how this differential modification of autoreactive T-cells by RTLs affects their fate and migration into the CNS remains to be established and will be addressed herein. Moreover, we specifically defined the functional changes induced by RTL treatment in the targeted encephalitogenic cell population that may account for recovery from EAE and CNS demyelination and axonal damage.

\section{Materials and Methods}

Animals. C57BL/6 male mice were obtained from The Jackson Laboratory (Bar Harbor, ME) at 6-7 weeks of age. The mice were housed in the Animal Resource Facility at the Portland Veterans Affairs Medical Center in accordance with institutional guidelines. The study was conducted in accordance with National Institutes of Health guidelines for the use of experimental animals, and the protocols were approved by the Institutional Animal Care and Use Committee.

Antigen. Mouse MOG-35-55 peptide (MEVGWYRSPFSRVVHLYRNGK) was synthesized by NeoMPS (San Diego, CA).

RTL construction, modification, and production. General methods for the design, cloning, and expression of RTLs have been described previously (Huan et al., 2004; Burrows et al., 1999; Fontenot et al., 2006). In brief, a series of murine MHC class II I-A ${ }^{\mathrm{b}}$-derived single-chain $\beta 1 / \alpha 1$ recombinant RTLs, termed rIA $^{\mathrm{b}}$ (RTL550), were constructed by sequential site-directed mutagenesis of rIA $^{\mathrm{q}}$ RTLs. The progenitor rIA ${ }^{\mathrm{q}}$ RTLs were constructed using mRNA isolated from the splenocytes of DBA1/ LacJ mice using an Oligotex Direct mRNA mini kit (Qiagen, Valencia, CA). cDNA of the antigen-binding/TCR recognition domain of murine I-A ${ }^{9}$ MHC class II $\beta 1$ and $\alpha 1$ chains was derived from mRNA using two pairs of PCR primers. The two chains were sequentially linked by a 5 aa linker (GGQDD) in a two-step PCR with NcoI and XhoI restriction sites being added to the $\mathrm{N}$ terminus of the $\beta 1$ chain and to the $\mathrm{C}$ terminus of the $\alpha 1$ chain, respectively, to create rIA $^{\mathrm{q}}$ (RTL560). "Empty" rIA $^{\mathrm{b}}$ (RTL550) was constructed by sequential site-directed mutagenesis using empty rIA ${ }^{\mathrm{q}}$ (RTL560) as a template. The 22 site-directed mutations required were inserted using eight primers containing the desired sequence changes and four consecutive mutagenesis cycles (QuikChange Multi site-directed mutagenesis kit; Stratagene, Cedar Creek, TX). An additional sequence encoding murine MOG-35-55 peptide (MEVGWYRSPFSRVVHLYRNGK) and a flexible linker (GSGSGSGSGSGSGS) was added to the $5^{\prime}$ end of the $\beta 1$ domain of rIA ${ }^{\mathrm{b}}$ RTL550 to form rIA ${ }^{\mathrm{b}} / \mathrm{MOG}$ (RTL551). The rIA ${ }^{\mathrm{b}} / \mathrm{MOG}$ insert was ligated into $\mathrm{pET} 21 \mathrm{~d}(+)$ vector and transformed into Nova blue Escherichia coli host (Novagen, Madison, $\mathrm{WI}$ ) for positive colony selection and sequence verification, and $\mathrm{rIA}^{\mathrm{b}}$ / MOG plasmid constructs were then transformed into the E. coli strain BL21(DE3) expression host (Novagen). Protein purification was as described previously (Huan et al., 2004; Burrows et al., 1999; Fontenot et al., 2006 ) with a $30-40 \mathrm{mg}$ yield of purified protein per liter of bacterial cell culture.

Induction of active EAE and treatment with RTL551. C57BL/6 mice were inoculated in the flanks with $0.2 \mathrm{ml}$ of emulsion containing $200 \mu \mathrm{g}$ of MOG-35-55 peptide and an equal volume of complete Freunds adjuvant (CFA) containing $4 \mathrm{mg} / \mathrm{ml}$ of heat-killed Mycobacterium tuberculosis. Mice were also given injections of 75 and $200 \mathrm{ng}$ of pertussis toxin (Ptx) intraperitoneally on days 0 and 2 relative to immunization. The mice were assessed for signs of EAE according to the following scale: 0 , normal; 1 , limp tail or mild hindlimb weakness; 2 , moderate hindlimb weakness or mild ataxia; 3, moderately severe hindlimb weakness; 4, severe hindlimb weakness or mild forelimb weakness or moderate ataxia; 5 , paraplegia with no more than moderate forelimb weakness; 6 , paraplegia with severe forelimb weakness or severe ataxia or moribund condition. At the onset of clinical signs of EAE (days 12-14 when the clinical scores were $\geq 2$ ), the mice were divided into two groups and treated with $100 \mu \mathrm{l}$ of $20 \mathrm{~mm}$ Tris- $\mathrm{HCl}$ as controls or with $100 \mu \mathrm{lof} 1 \mathrm{mg} / \mathrm{ml}$ RTL551 intravenously along with antihistamine for $5 \mathrm{~d}$. Mice were monitored for changes in disease score until they were killed for ex vivo analyses.

Induction of passive EAE. To study the migration and fate of encephalitogenic cells after RTL treatment, green fluorescent protein (GFP) mice on the C57BL/6 background were immunized with $200 \mu \mathrm{g}$ of MOG$35-55$ in $400 \mu \mathrm{g}$ of CFA. Ten days after immunization, lymph nodes and spleens were harvested and cultured in vitro in the presence of $25 \mu \mathrm{g} / \mathrm{ml}$ MOG-35-55 peptide in stimulation medium containing 10\% FBS for $48 \mathrm{~h}$. Cells were then washed, and 50 million activated cells were injected intraperitoneally into wild-type C57BL/6 mice along with 75 and $200 \mathrm{ng}$ of Ptx injected intraperitoneally on days 0 and 2 relative to cell transfer. The mice were assessed daily for signs of EAE according to the scale presented above. At the onset of clinical signs of EAE (days 7-12 when the clinical scores were $\geq 2$ ), the mice were divided into two groups and treated with $100 \mu \mathrm{l}$ of $20 \mathrm{~mm}$ Tris- $\mathrm{HCl}$ as controls or with $100 \mu \mathrm{l}$ of 1 $\mathrm{mg} / \mathrm{ml} \mathrm{RTL551} \mathrm{intravenously} \mathrm{along} \mathrm{with} \mathrm{antihistamine} \mathrm{for} 5 \mathrm{~d}$. Mice were monitored for changes in disease score until they were killed for $e x$ vivo analyses.

Effect of RTL treatment on cytokine production by encephalitogenic cells. Single-cell suspensions of spleens from vehicle and RTL551 treatment groups were prepared by homogenizing the tissue through a fine mesh screen. GFP-expressing and GFP-nonexpressing cells were further sorted using a FACSVantage (BD Biosciences, Mountain View, CA). Sorted cells had a purity of $99.9 \%$. Four million GFP-expressing and GFPnonexpressing cells from each control and RTL-treated group were cultured in the presence of $25 \mu \mathrm{g}$ of MOG-35-55 peptide in a 24-well tissue culture plate for $48 \mathrm{~h}$. Culture supernatants were assessed for cytokine levels using a Luminex Bio-Plex cytokine assay kit (Bio-Rad, Richmond, CA) following the manufacturer's instructions. The following cytokines were determined in a single assay in three separate experiments: IL-1 $\beta$, interferon $\gamma(\mathrm{IFN} \gamma)$, tumor necrosis factor $\alpha$ (TNF $\alpha)$, IL-2, IL-4, IL-10, IL-17, IL-5, IL-6, IL-12 and IL-13. Alternatively, total RNA was isolated from sorted GFP + cells using the RNeasy mini-kit protocol (Qiagen) and converted to cDNA using oligo(dT), random hexamers, and Superscript RT II enzyme (Invitrogen, Grand Island, NY). Real-time PCR for IL-17 and TNF $\alpha$ was performed using Quantitect SYBR Green PCR master mix (Qiagen) and primers (synthesized by Applied Biosystems, Foster City, CA). Reactions were conducted on the Applied Biosystems Prism 7000 Sequence Detection System using the listed primer sequences ( $5^{\prime}-$ $3^{\prime}$ ) to detect the following genes: $L 32$ (forward, GGAAACCCAGAGGCATTGAC; reverse, TCAGGATCTGGCCCTTGAAC); IL-17 (forward, CCCAGGGTCAGGAAGACA; reverse, CCGAAGGACCAGGATTTCT); TNF $\alpha$ (forward, CAGCCGATGGGTTGTACCTT; reverse, GGCAGCCTTGTCCCTTGA).

Detection of GFP-expressing cells in the spleens and spinal cords of mice with passive EAE. At different time points, animals were perfused with $0.9 \%$ saline followed by cold $4 \%$ paraformaldehyde. Tissues were postfixed in $4 \%$ paraformaldehyde overnight at $4^{\circ} \mathrm{C}$. Spleens and spinal cords were removed and transferred into $30 \%$ sucrose at $4^{\circ} \mathrm{C}$ for cryoprotection. Transverse segments of the lumbar region of the spinal cord were frozen in OCT compound (Tissue-Tek; Electron Microscopy Sciences, Hatfield, PA). Ten micrometer frozen sections were air dried, rinsed in PBS, coverslipped with fluoromount G, and examined under a fluorescent microscope for visualization of GFP-expressing cells. Alternatively, lumbar spinal cord sections were also stained with biotinylated anti-GFP (Abcam, Cambridge, MA), and cells expressing GFP were identified with streptavidin HRP conjugate followed by counterstaining of the tissue with hematoxylin.

Detection of apoptotic cells in the spinal cords of mice with EAE. Apoptotic cells in the spinal cords were detected in situ by the terminal deoxynucleotidyl transferase-mediated biotinylated UTP nick end labeling (TUNEL) assay according to the manufacturer's instructions (Roch Di- 
agnostics, Mannheim, Germany). Quantification of apoptotic cells was done by counting the number of positive cells in randomly chosen, noncontiguous high-power $(40 \times)$ fields until a minimum total of 400 cells were counted.

Chemokine and receptor array. Quantitative mRNA expression analysis of 84 chemokines and their receptors in spinal cords of mice was performed with the mouse chemokine and receptor $\mathrm{RT}^{2}$ profiler PCR array (SuperArray Bioscience Corporation, Frederick, MD). Total RNA was isolated from spinal cords of individual mice with active EAE from the vehicle- and RTL551-treated groups $(n=3)$ using the RNeasy mini kit protocol (Qiagen). Equal amounts of RNA from each sample were then converted to cDNA using oligo(dT), random hexamers, and Superscript RT II enzyme (Invitrogen Life Technologies). cDNAs from each experimental condition were pooled, and PCR array analysis was performed according to the manufacturer's protocol with the $\mathrm{RT}^{2}$ Real-Time SYBR Green PCR Master Mix (SuperArray Bioscience Corporation). PCRs were performed using $\mathrm{RT}^{2}$ profiler PCR array APMM-022 (mouse chemokines and receptors) on the Applied Biosystems 7500 Fast using $\mathrm{RT}^{2}$ Real-Time SYBR Green PCR master mix. The total volume of the PCR was $25 \mu$. The thermocycler parameters were $95^{\circ} \mathrm{C}$ for $10 \mathrm{~min}$, followed by 40 cycles of $95^{\circ} \mathrm{C}$ for $15 \mathrm{~s}$ and $60^{\circ} \mathrm{C}$ for $1 \mathrm{~min}$. mRNA expression of each gene was normalized using the expression of glyceraldehyde-3phosphate dehydrogenase as a housekeeping gene and compared with the data obtained from with the control group (vehicle treated) according to the $2^{-\mathrm{DDCT}}$ method (Livak and Schmittgen, 2001). The results were confirmed by quantitative reverse transcription (RT)-PCR on the individual RNA samples.

Immunohistochemistry. Four mice from the vehicle- and RTL551treated groups with active EAE were perfused with $0.9 \%$ saline followed by cold $4 \%$ paraformaldehyde. Spinal cords were removed and postfixed in $4 \%$ paraformaldehyde overnight for $2 \mathrm{~h}$. Four sections of the lumbar spinal cord region per mouse were stained with each antibody. The sections were permeabilized with $0.2 \%$ Triton X-100 in PBS for 45 min followed by blocking with fish skin gelatin $(0.5 \%)$ and BSA (3\%) for $2 \mathrm{~h}$ at room temperature. The sections were then incubated with primary antibody in blocking solution overnight at $4^{\circ} \mathrm{C}$. The next day, the sections were washed with PBS and incubated with the appropriate secondary antibody for $2 \mathrm{~h}$ at room temperature. After washing with PBS, the sections were mounted in Prolong Gold anti-fade and visualized on a Nikon (Tokyo, Japan) laser-scanning confocal microscope. Primary antibodies were as follows: anti-VCAM-1, anti-ICAM-1 (for cell adhesion molecules), and anti-vWF [endothelial cell marker (Chaudhary et al., 2006)]. Primary and secondary antibodies were used at a dilution of 1:25 and $1: 200$, respectively. White matter of the dorsal, lateral and ventral spinal cord was photographed under 40x magnification (for determining the percentage of area covered by an antibody stain) and 60x magnification (for co-localization of immunofluorescence) on a laser scanning confocal microscope. Digital images were saved in 12-bit TIFF format $(1024 \times$ 1024 pixels). Data were generated from 20 raw images ( 4 mice each in naive, vehicle and RTL551 treatment groups). All image processing and analysis was done using BITPLANE Imaris software version 5.7.1 (Switzerland). Background fluorescence was determined on the sections incubated only with the secondary antibody. An average fluorescence was also determined from several unlabeled regions of the spinal cords. The threshold was set two times above the background signal/noise. For colocalization of immunofluorescence, signal threshold values in the two images to be analyzed were set against the appropriate controls. Threshold pixels in the source A image (for example VCAM-1) that were also the threshold pixels in the source B image (vWF) were measured. Data were exported to an excel spreadsheet. All the images were taken with exactly the same acquisition settings and all the data analyses were done in a blinded manner.

Statistical analysis. Statistical difference of clinical EAE scores between vehicle and treatment groups was determined by the Mann-Whitney $U$ test. Differences in cytokine levels were evaluated by Student's $t$ test. $p$ values $\leq 0.05$ were considered significant.

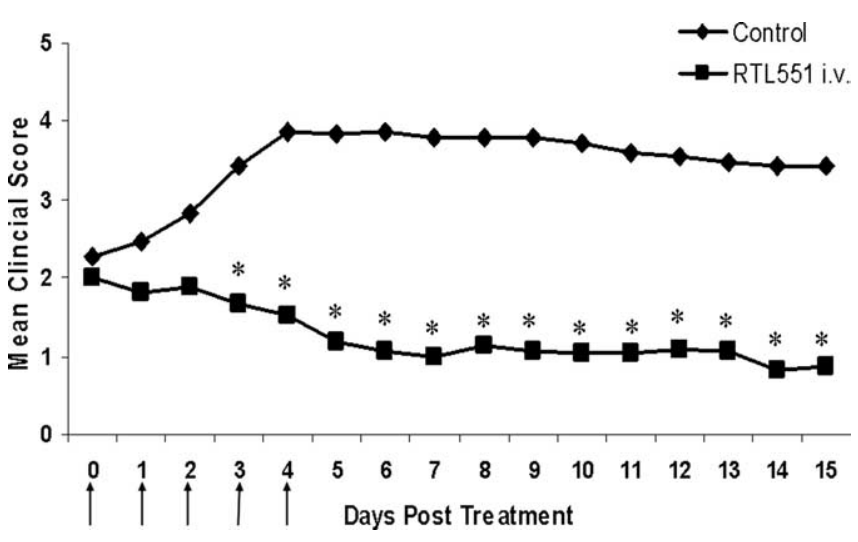

Figure 1. Intravenous administration of RTL551 treats EAE in C57BL/6 mice. C57BL/6 male mice were immunized with MOG-35-55 peptide. At disease onset (day 11), mice were treated daily with vehicle or $0.1 \mathrm{mg}$ of RTL551 intravenously for $5 \mathrm{~d}$. Mice were scored as outlined in Materials and Methods. Data presented are the mean of three experiments for each group, with 8-10 mice per group. Significant differences between control and treated groups $\left({ }^{*}\right)$ were determined using the Mann-Whitney $U$ test $\left({ }^{a}, p<0.05\right)$.

\section{Results}

\section{RTL551 reverses clinical signs of EAE}

In previous studies, we demonstrated that RTLs containing encephalitogenic peptides linked to the outer two domains of the restricting MHC Class II molecules could reverse clinical signs of EAE (Offner et al., 2005). Using the same approach, we constructed a new RTL containing the $\alpha 1$ and $\beta 1$ domains of the I-A ${ }^{\mathrm{b}}$ Class II molecule covalently linked to the encephalitogenic MOG-35-55 peptide, a construct termed RTL551, and evaluated its therapeutic efficacy and mechanism of action in chronic EAE in C57BL/6 mice. At onset of clinical signs of actively-induced EAE (d12-14), mice were treated with RTL551 intravenously for $5 \mathrm{~d}$. The RTL treatment was very effective in reducing the clinical severity of EAE and arresting disease progression throughout the observation period that lasted for $20 \mathrm{~d}$ post-immunization. The vehicle-treated mice showed a cumulative disease index (CDI) of $39.8 \pm 6.0$ whereas the RTL-treated mice had a significantly reduced CDI of $17.1 \pm 4.9$ ( $p<0.01$, Fig. 1$)$. The peak disease score of RTL551-treated mice also was significantly lower than vehicle treated mice $(5.0 \pm 0.0$ vs. $2.4 \pm 0.3 ; p<0.05$, Fig. 1$)$. The therapeutic effect of RTL551 was highly reproducible in three separate experiments.

\section{Localization of passively transferred GFP expressing MOG- 35-55 specific T-cells in CNS after treatment with RTL551}

To study the fate and migration of encephalitogenic cells after RTL551 treatment, we transferred 50 million GFP-expressing cells specific for MOG-35-55 peptide into wild type C57BL/6 recipients. At disease onset $(\mathrm{d} 7-12)$ mice were treated with RTL551 intravenous for $5 \mathrm{~d}$. As with actively induced EAE, the RTL treatment nearly abolished clinical signs of passive EAE, reducing CDI scores from $49.8 \pm 7.1$ for control mice to $14.8 \pm$ $7.8(p<0.05$, Fig. 2$)$. The peak disease score was also significantly lower in the RTL551-treated mice $(2.1 \pm 0.38$ vs. $5 \pm 0$ in controls; $p<0.01$, Fig. 2). Spleens were harvested $19 \mathrm{~d}$ post immunization and the cells were subjected to sorting for the recovery of GFP expressing and GFP non-expressing populations. Four million sorted cells (GFP expressing as well as GFP non-expressing) from each group were cultured in the presence of APC and $25 \mu \mathrm{g}$ MOG-35-55 peptide for $48 \mathrm{~h}$ followed by cytokine detection in the culture supernatants. Recently it has been shown that IL- 


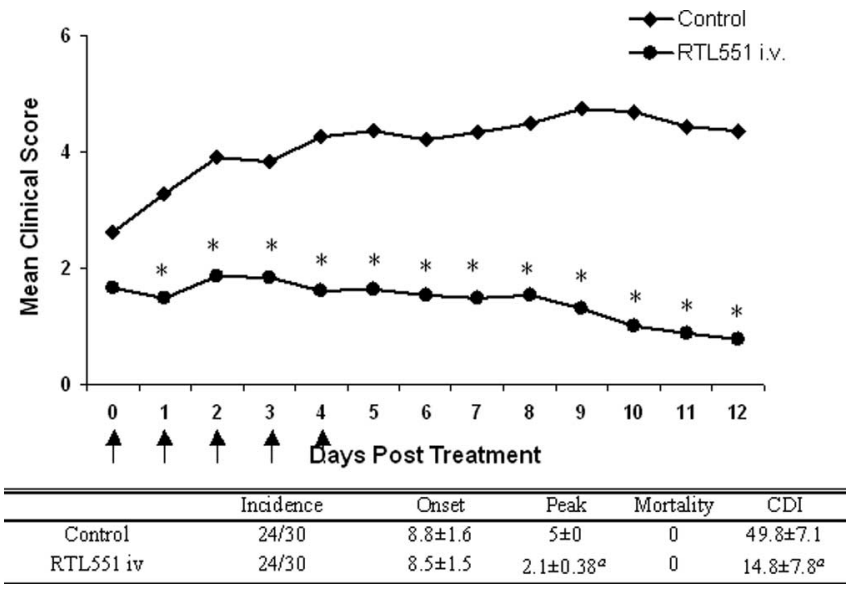

Figure 2. RTL 551 treats passively induced EAE. Fifty million MOG-35-55 specific cells were injected intraperitoneal into C57BL/6 mice. At disease onset (day 8) mice were treated daily with vehicle or $0.1 \mathrm{mg}$ of RTL551 intravenously for $5 \mathrm{~d}$. Mice were scored as outlined in Materials and Methods. Data presented are the mean of three experiments for each group, with 8-10 mice per group. Significant differences between control and treated groups $(*)$ were determined using the Mann-Whitney $U$ test $\left({ }^{a}, p<0.05\right)$.
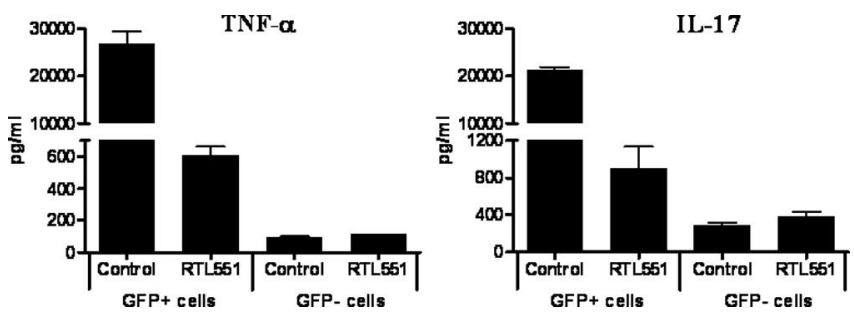

Figure 3. Cytokine production in the spleen of RTL- vs. vehicle-treated mice with EAE. Fifty million MOG-35-55 specific cells from GFP mice were injected intraperitoneal into C57BL/6 mice. Mice were killed one day after the last injection of buffer or RTL and mononuclear cells from spleen were sorted to recover GFP expressing and GFP non-expressing cells. Sorted cells from each group were cultured in vitro with $25 \mu \mathrm{g}$ of MOG-35-55 peptide. Supernatants were harvested after $48 \mathrm{~h}$ and assayed for cytokine production using Bio-Plex cytokine assay kit, as described in Materials and Methods. Significant differences between control and experimental groups were determined using Student's $t$ test $\left({ }^{*}, p<0.05\right)$. Data are presented as the mean \pm SD of three replicate cultures from pooled cells, and are representative of three experiments.

$17 \mathrm{KO}$ mice have delayed onset and reduced severity of EAE (Komiyama et al., 2006). Furthermore, adoptively transferred T-cells from IL-17KO mice are not efficient for EAE induction (Komiyama et al., 2006), indicating a key role for IL-17 in EAE. Interestingly in our assay, IL-17 was secreted almost exclusively by transferred encephalitogenic GFP expressing cells, with negligible amounts secreted from bystander (GFP non-expressing) cells (Fig. 3, right panel). Moreover, IL-17 was significantly down-regulated in the cells from RTL551-treated mice as compared with control mice ( $p<0.05$, Fig. 3 , right panel). Another pro-inflammatory cytokine, TNF- $\alpha$, which also was almost exclusively contributed by the transferred encephalitogenic cells, was greatly reduced with RTL treatment ( $p<0.05$, Fig. 3$)$. Effect of RTL treatment on IL-17 and TNF $\alpha$ gene expression in MOG35-55 specific cells was also confirmed by real-time PCR. Expression of each gene was calculated relative to the expression of housekeeping gene, L32. mRNA analysis of the sorted encephalitogenic cells also confirmed the significantly reduced relative expression of IL-17 (undetected in RTL551 treated mice) and TNF $\alpha$ (2.5 fold reduction in RTL551 treated mice) from RTL551 treated as compared with vehicle treated mice. It is noteworthy that IL-17 was the major cytokine detected in the culture super-
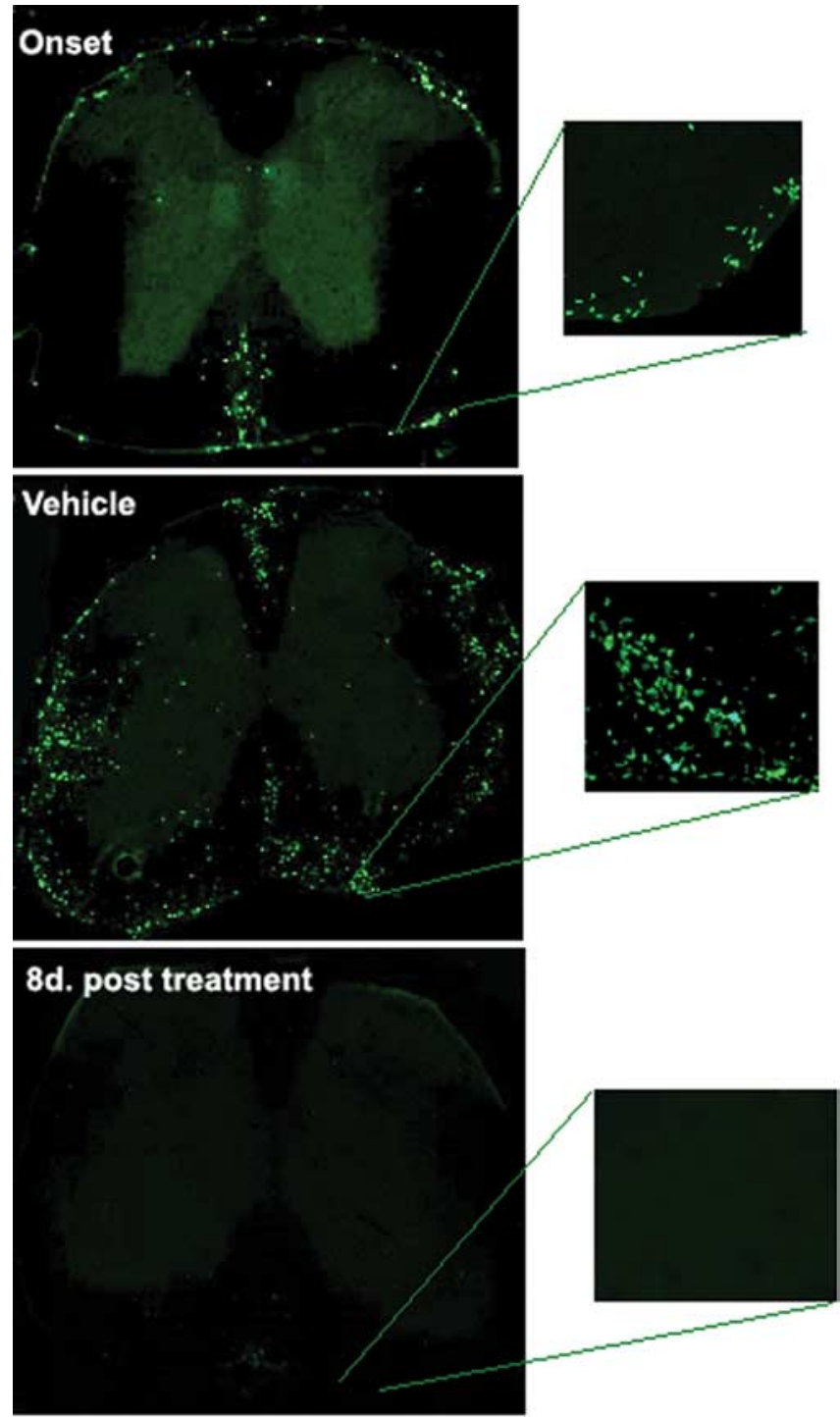

Figure 4. Fixed, frozen spinal cord sections from mice, as visualized under fluorescence microscope, either at onset (top), or treated with vehicle (middle) or RTL (bottom). Spinal cords of RTL treated mice showed remarkable reduction of GFP expressing MOG-235-55 specific cells $3 \mathrm{~d}$ post treatment completion (bottom as compared with middle). Magnification top, middle and bottom $\times 4$; inset $\times 20$.

natants of cells from the organ stimulation (data not shown). This suggests that IL-17 might be the major cytokine contributing to the encephalitogenicity of the cells and, therefore, its down-regulation by RTL551 could account for loss of encephalitogenic activity. Most of the remaining cytokines studied, including IFN- $\gamma$, were equally produced by GFP expressing (transferred) and GFP non-expressing (host) cells and were not significantly modulated by RTL551 treatment.

Additionally we evaluated the effect of RTL treatment on the infiltration of encephalitogenic cells in the spinal cords of mice with passive EAE (Fig. 4). Mice were killed before and $8 \mathrm{~d}$ after the initiation of RTL treatment and spinal cords were processed for the visualization of GFP expressing cells. Infiltration of encephalitogenic cells in the spinal cords was greatly reduced after only three RTL treatments and the GFP expressing cells were completely absent in the spinal cords upon treatment completion (not shown). This dramatic effect on the infiltration of cells in the CNS was observed in three separate experiments. Moreover, the 


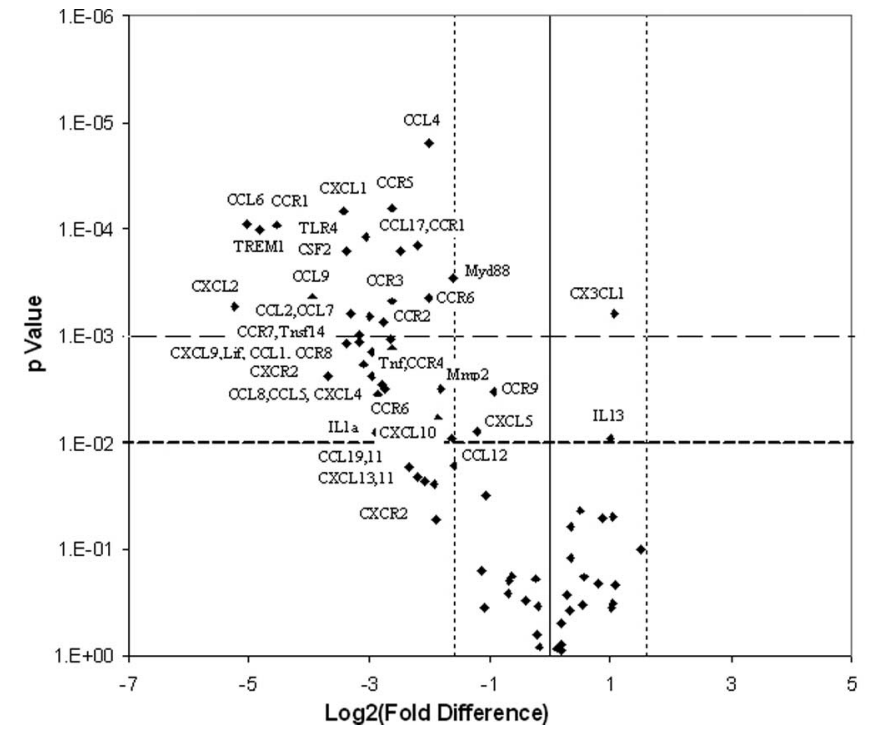

Figure 5. Volcano plot between Log2 fold change (for chemokines and receptors in RTLtreated vs. control groups) vs. p value. (57BL/6 male mice were immunized with MOG-35-55 peptide/CFA/Ptx and treated with RTL551 intravenously at disease onset for $5 \mathrm{~d}$. CDNA prepared from the spinal cords of mice were subjected to $\mathrm{RT}^{2}$ profiler $\mathrm{PCR}$ array for mouse chemokines and receptors, as described in Materials and Methods. The results of the pooled mRNA samples were confirmed by running the RT-PCR array on individual samples (3 mice per group). Significant differences between control and experimental groups were determined using the MannWhitney $U$ test. Vertical dotted lines represent a threefold change in gene expression (threshold for fold difference).

reduction in inflammatory infiltrates was reflected in the clinical scores of the disease. The RTL551-treated mice had a negligible score of 0.5 starting on the fourth day of treatment. RTL treatment not only reduced the number of already infiltrated cells, but was also capable of blocking the entry of any more inflammatory cells into the CNS, since the spinal cords of the treated mice were completely lacking of the encephalitogenic cells $3 \mathrm{~d}$ after the final injection of RTL551 (Fig. 4A and C). To more precisely localize the transferred encephalitogenic cells in the spinal cord of treated mice, lumbar sections of the spinal cord were also stained with biotinylated anti-GFP and counter stained with hematoxylin. We found that the GFP expressing cells were restricted to the subarachnoid space in the spinal cords of the RTL551-treated group (data not shown) and therefore, were unavailable to contribute to CNS damage. In contrast, GFP expressing cells in control CNS tissue were distributed throughout the parenchyma of the white matter and along the perivascular space in the spinal cord (not shown). Since the already infiltrated green cells (Fig. 4, mice at onset) were also absent in the spinal cord of RTL551-treated mice, we questioned whether the transferred cells might be undergoing apoptosis with RTL treatment. Thus, TUNEL staining was performed on the spinal cords of control and treated mice at 1 and $3 \mathrm{~d}$ after RTL551 treatment. However, we did not observe any increase in cells undergoing apoptosis in the spinal cords of the RTL551-treated group compared with control mice (data not shown).

\section{Effects of RTL551 therapy on expression of chemokines/receptors in CNS tissue}

The striking reduction of infiltrating cells into the CNS suggested that the RTL treatment created an unfavorable environment for the encephalitogenic cells such that they were not able to sustain themselves, and no more cells could gain entry in the parenchyma
Table 1. Chemokines and receptors significantly modulated by RTL551 treatment in the spinal cords of $\mathrm{C57BL} / 6$ mice with MOG-35-55-induced EAE

\begin{tabular}{|c|c|c|}
\hline Chemokine/receptor & $\begin{array}{l}\text { Fold upregulation } \\
\text { or downregulation }\end{array}$ & $p$ value \\
\hline CCL1/CCR8 & $-6 /-8$ & $<0.01$ \\
\hline CCL2/CCR2 & $-10 /-7$ & $<0.001$ \\
\hline $\mathrm{CCL} 4 / \mathrm{CCR} 5$ & $-4 /-6$ & $<0.001$ \\
\hline CCL5/CCR1, CCR3, CCR5 & $-7 /-23,-6,-6$ & $<0.01 /<0.00$ \\
\hline CCL6/CCR1 & $-33 /-23$ & $<0.001$ \\
\hline CCL7/CCR1, CCR2, CCR3 & $-8 /-23,-7-6$ & $<0.001$ \\
\hline CCL8/CCR3 & $-7 /-6$ & $<0.01 /<0.00$ \\
\hline CCL9/unknown & -15 & $<0.001$ \\
\hline CCL17/CCR4 & $-8 /-8$ & $<0.001 /<0.0$ \\
\hline CCL19/CCR7 & $-5 /-9$ & $<0.05 /<0.00$ \\
\hline CXCL1/CXCR2 & $-11 /-13$ & $<0.001 /<0.0$ \\
\hline CXCL2/CXCR2 & $-38 /-13$ & $<0.001 / 0.01$ \\
\hline CXCL4/unknown & -7 & $<0.01$ \\
\hline CXCL5/CXCR2 & $-2 /-13$ & $<0.01$ \\
\hline CXCL9, CXCL10 & $-9,-3$ & $<0.01$ \\
\hline CXCL12/CXCR4 & $-2 /-6$ & $<0.05 /<0.00$ \\
\hline CCR6 & -4 & $<0.001$ \\
\hline CCR9 & -2 & $<0.01$ \\
\hline CCR12 & -6 & $<0.001$ \\
\hline CXCR6 & -4 & $<0.01$ \\
\hline GMCSF & -10 & $<0.001$ \\
\hline IL-1a & -8 & $<0.01$ \\
\hline LIF & -10 & $<0.01$ \\
\hline MMP2 & -4 & $<0.01$ \\
\hline Myd88 & -3 & $<0.001$ \\
\hline TLR4 & -8 & $<0.001$ \\
\hline $\operatorname{TNF} \alpha$ & -9 & $<0.01$ \\
\hline TREM1 & -29 & $<0.001$ \\
\hline CX3CL1 & 2 & $<0.001$ \\
\hline IL-13 & 2 & $<0.01$ \\
\hline
\end{tabular}

of the white matter. It has been shown previously that entry of inflammatory cells into CNS parenchyma is essential for EAE induction (Gimenez et al., 2004), a process that is regulated by blood brain barrier disruption and increased levels of chemokines and chemokine receptors. We thus evaluated spatial expression of 84 chemokine/receptor genes in the spinal cords of control and RTL551-treated mice (Fig. 5 and Table 1). Of particular interest were the chemokines CXCL2 and CCL6 which were 41 $(p<0.001)$ and $33(p<0.001)$ fold down-regulated, respectively, in RTL551 treated spinal cords. CXCL2 (MIP-2) has been shown to be primarily associated with reactive astrocytes and its nanomolar concentrations markedly up-regulate expression of CCL2/MCP1 and RANTES (Luo et al., 2000). Thus, stimulating the chemokine cascade in astrocytes in response to CXCL2 likely contributes to persistent mononuclear cell infiltration in the spinal cords. It is thus of importance to note that both MCP1 and RANTES were also respectively down-regulated $10-(p<0.001)$ and 7- $(p<0.01)$ fold in RTL551-treated spinal cords. CCL6 is produced primarily by microglia and macrophages and acts in an autocrine manner as a potent chemo-attractant of macrophages in CNS (Asensio et al., 1999; Kanno et al., 2005). CXCR2, the receptor for CXCL2, was down-regulated $13(p<0.01)$ fold in the RTL551-treated spinal cords. This receptor is expressed on leukocytes migrating from the blood, including neutrophils and macrophages, and has been shown to be significantly increased during the first and second disease attacks in EAE (Glabinski et al., 2000). CCR1, receptor for CCL6 and down regulated 23 ( $p<$ 0.001 ) fold in RTL551 treated spinal cords, is down regulated in CNS during remission of MOG-induced EAE in rats leading to 
diminished inflammation and demyelination (Eltayeb et al., 2007). The importance of CCR1 in the recruitment of leukocytes during EAE has been demonstrated in CCR1KO mice which develop EAE with reduced severity and incidence (Rottman et al., 2000).

Other MIP-1 family chemokines like CCL4 and CCL9 were also down-regulated in the RTL551-treated group (Table 1). Among other chemokines, CXCL1 and its receptor CXCR2 were down-regulated 11 $(p<0.001)$ and $13(p<0.01)$ fold, respectively in RTL treatment group. CXCL1 has been shown to be selectively produced by parenchymal tissues and has been found to be upregulated in EAE (Glabinski et al., 1999). As mentioned above, CCL2/MCP1, produced by many cell types including resident glial cells and astrocytes, was 10 ( $p<0.001)$ fold downregulated, and its receptor, CCR2, was 7 $(p<0.001)$ fold down-regulated in RTL551-treated spinal cords. CCL1/ MCP1 promotes leukocyte adhesion to brain microvasculature, and its expression in CNS positively correlates with the degree of inflammation and severity of EAE, being crucial for the transmigration of CCR2 positive cells (microglia, macrophages and perivascular mononuclear cells) into the parenchyma (Mahad and Ransohoff, 2003). Moreover, lack of CXCL1 may prevent recruitment of inflammatory cells to the CCL2 expressing sites (Huo et al., 2001). Recently, CCR2 deficient mice were found to be resistant to MOG-induced EAE (Izikson et al., 2000). These mice lacked inflammatory infiltrates and failed to upregulate RANTES, MCP-1, IP10, CCR1, CCR2 and CCR5 in the CNS. Of note is one other chemokine, leukemia inhibitory factor (LIF), which was downregulated $10(p<0.01)$ fold in RTL551-treated spinal cords. LIF was recently shown to play a pro-inflammatory role after injury to the nervous system, being required for the full activation of astrocytes and microglia/macrophages, and providing a necessary signal for the influx of peripheral immune cells in the spinal cord (Kerr and Patterson 2004). Taken together the data suggest that due to the loss of a chemotactic gradient, the inflammatory infiltrates are not able to sustain themselves in the spinal cords of RTL551-treated mice, leading to a dramatic reduction of cellular infiltrates.

\section{RTL551 treatment inhibits expression of VCAM1 and ICAM1 by CNS vascular endothelial cells}

Along with the chemokine gradient, up-regulation of cell adhesion molecules, VCAM1 and ICAM1, on CNS endothelial cells and astrocytes, is required for the entry and persistence of inflammation in the CNS parenchyma that contribute to chronic EAE. To study the effect of RTL551 treatment on the expression of VCAM1 and ICAM1, mice were immunized with MOG-35-55/ CFA/Ptx to induce EAE, and half of the mice were treated with RTL551 intravenously for $5 \mathrm{~d}$ after onset of clinical signs. Upon completion of RTL treatment, mice were perfused with ice cold
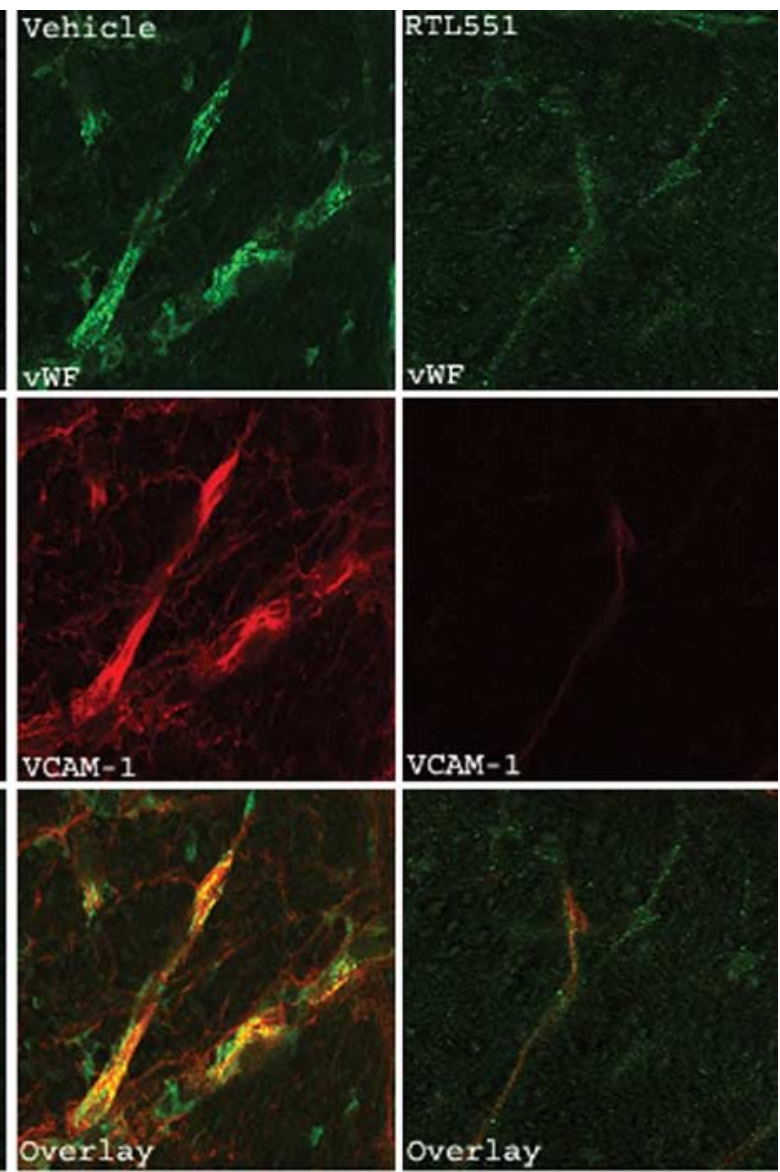

Overlay

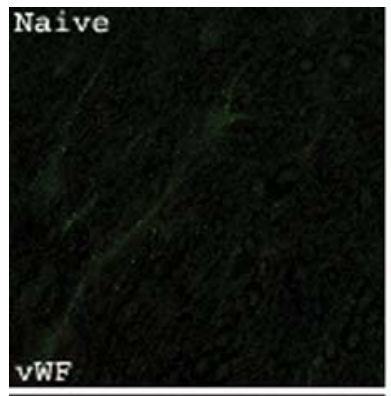

Figure 6. RTL551 treatment down-regulates VCAM-1 expression on CNS vascular endothelial cells. Representative spinal cord sections were taken one day after the fifth daily injection of RTL551. Fifty micron thick sections from the lumbar region of the spina cord were double labeled with anti-VCAM-1 and anti-vWF. Sections were examined with a confocal microscope with a 60X objective. Also note increased expression of VCAM-1 and vWF in EAE vs. naive spinal cord sections.

PBS followed by PFA and the spinal cords were processed for dual immunolabeling with anti-vWF (an endothelial cell marker) in combination with anti-VCAM-1 (Fig. 6) or anti-ICAM-1 (Fig. 7 ). The percentage of area covered by antibody stain for VCAM-1, as calculated by thresholding the image, was $27.8 \%$, $2.9 \%$ and $3.4 \%$ in mice with vehicle treatment, naive and RTL551 treatment respectively $(p<0.01)$. Similarly, the area of staining for ICAM-1 in mice with vehicle treatment, naive and RTL551 treatment was $18.7 \%, 1.5 \%$ and $2.7 \%$ respectively $(p<0.05)$. The average co-localized area (normalized to the total area) of VCAM-1 over vWF was $2.4 \%$ in the vehicle-treated group as compared with $0.01 \%$ and $0.03 \%$ in naive and RTL551-treated groups, respectively (vehicle vs. RTL551; $p<0.01$ ). Similarly, the average area of co-localization between ICAM-1 over vWF was $0.93 \%$ in the vehicle-treated group as compared with $0.01 \%$ and $0.02 \%$ in naive and RTL551-treated groups, respectively (vehicle vs. RTL551; $p<0.01$ ). Therefore, the data clearly demonstrate that not only was the expression of VCAM-1 and ICAM-1 increased on CNS vascular endothelial cells in EAE, but also that RTL551 treatment significantly down-regulated these adhesion molecules to near background levels.

\section{Discussion}

We have previously shown that RTL342 could reverse MOG35-55 peptide-induced EAE in DR2 Tg mice, and that RTL401 could reverse clinical and histological signs of PLP-139-151 peptide-induced EAE in SJL/J mice in a peptide-specific manner 

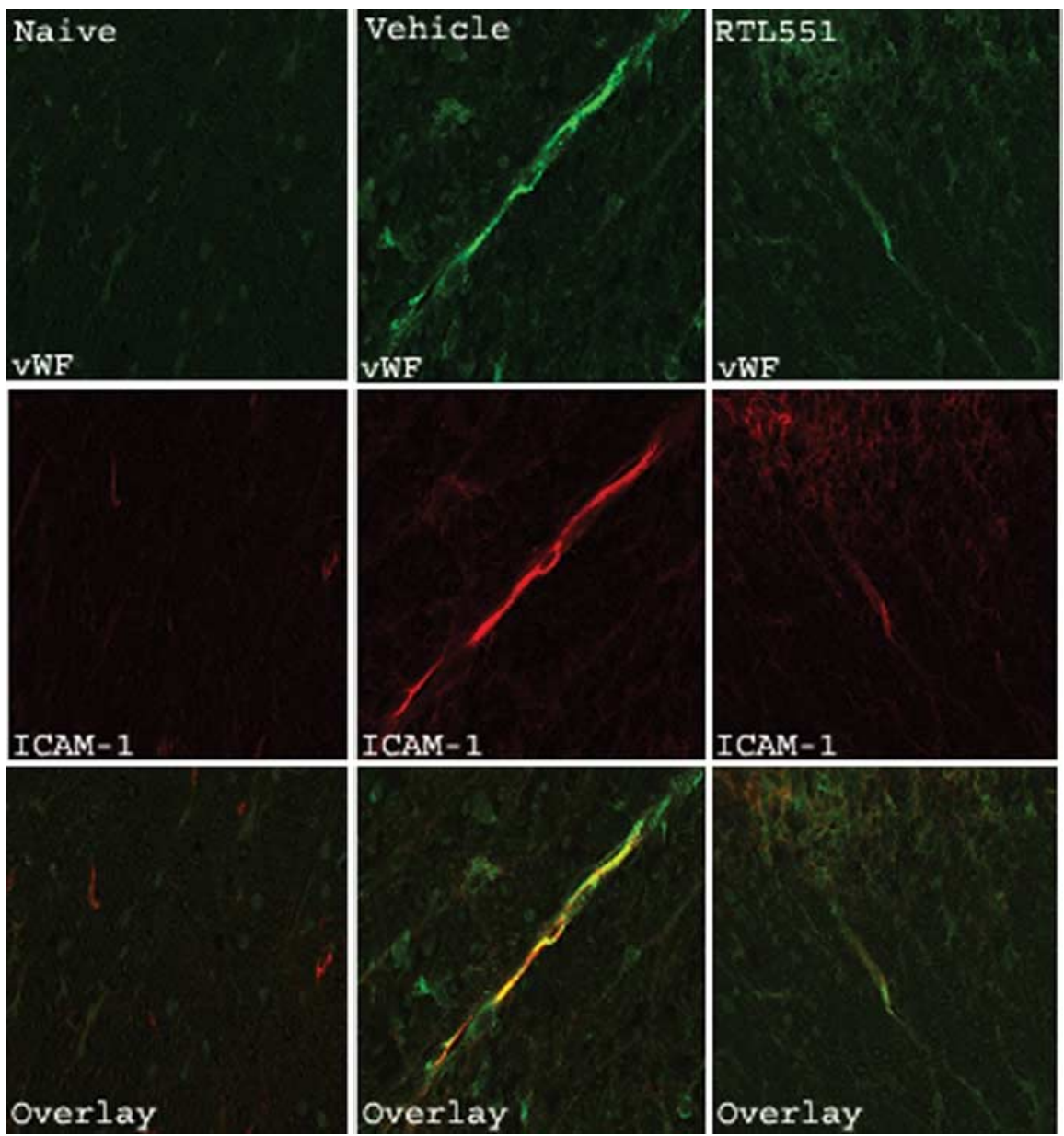

Figure 7. RTL551 treatment down-regulates ICAM-1 expression on CNS vascular endothelial cells. Representative spinal cord sections were taken one day after the fifth daily injection of RTL551. Fifty micron thick sections from the lumbar region of the spinal cord were double labeled with anti-ICAM-1 and anti-vWF. Sections were examined with a confocal microscope with a 60X objective. Also note increased expression of ICAM-1 and vWF in EAE vs. naive spinal cord sections.

(Vandenbark et al., 2003; Huan et al., 2004; Offner et al., 2005). In this study, we further explored the efficacy and mechanism of action of RTL551 that was specifically designed to treat chronic EAE induced by MOG-35-55 peptide in C57BL/6 mice. The data presented above clearly demonstrate that RTL551 is very effective in ameliorating the clinical signs of both active and passive MOGinduced EAE by systemic down-regulation of pathogenic cytokines and inhibition of cellular infiltration into the CNS through modulation of chemokines/receptors and expression of VCAM and ICAM on vascular endothelial cells.

To directly study the effect of RTL treatment on the encephalitogenic cells, 50 million MOG-specific cells from GFP + mice were transferred to wild type C57BL/6 mice. This model enabled us to selectively study the functional differences in recovered GFP expressing disease-causing cells vs. GFP non expressing host recruited splenocytes in response to RTL treatment. The most important conclusions from this study were 1) that both IL-17 and $\mathrm{TNF} \alpha$, known pathogenic cytokines, were contributed mainly by encephalitogenic cells, and 2) that treatment of the mice with RTL551 strongly reduced secretion of these cytokines. It has now been established that IL-17 producing effector T-cells are associated with many chronic inflammatory and autoimmune diseases including EAE, arthritis, colitis and asthma (Bettelli et al., 2007). Its importance in EAE is underscored by the fact that IL-17KO mice developed EAE with reduced severity and delayed onset
(Komiyama et al., 2006). Moreover, neutralizing antibody to IL-17 abrogated the progression of clinical EAE (Park et al., 2005; Langrish et al., 2005; Chen et al., 2006). This cytokine has also been detected in the sera and target tissue of patients with MS (Bettelli et al., 2007). Its down-regulation in RTL-treated mice might render activated cells less encephalitogenic. In accordance with this idea, MOG-35-55 specific T-cells from IL$17 \mathrm{KO}$ mice have been shown to be inefficient in inducing EAE in WT recipient animals (Komiyama et al., 2006). The contribution of TNF $\alpha$ in EAE has long been recognized. TNF $\alpha$ deficient mice developed EAE with delayed onset and reduced severity (Matejuk et al., 2002). Like IL-17, neutralizing antibodies to TNF $\alpha$ also ameliorated clinical and histological signs of EAE (Ruddle et al., 1990; Selmaj et al., 1991a; Baker et al., 1994). TNF $\alpha$ might be important in MS, since it has been found to be expressed in high levels in brain tissues, serum, cerebrospinal fluid and infiltrating as well as residual cells of CNS from MS patients (Selmaj et al., 1991b; Rentzos et al., 1996; Martino et al., 1997; Bitsch et al., 2000). Recent reports have also suggested that TH17 cells also produce TNF $\alpha$ along with IL-17A and IL17F (Bettelli et al., 2007; Langrish et al., 2005). In our cytokine assay also, GFP expressing encephalitogenic cells seemed to be the common source of IL-17 and $\mathrm{TNF} \alpha$, since both of these cytokines were secreted by transferred cells and to a much less extent by host cells. This is the first time that we have demonstrated the effect of RTL therapy on antigen specific cells in EAE. However, whether RTLs bind directly to these cells for modification of their pathogenic potential remains to be established.

With the GFP transfer experiment, we could also study the migration of encephalitogenic cells after treatment with RTL551. This model makes it possible to differentiate between encephalitogenic cells from bystander cells in the CNS and therefore we could study the treatment effect directly on the disease causing cells. We observed a dramatic reduction of encephalitogenic cells in the spinal cords of mice $3 \mathrm{~d}$ after the last treatment with RTL551. This was similar to our previous treatment experiment with a DR2 RTL covalently linked to the encephalitogenic MOG35-55 peptide (Vandenbark et al., 2003) that markedly reduced inflammatory lesions and demyelination in the spinal cord of DR2 transgenic mice with EAE. Concomitant with this, treatment with the RTL401 construct resulted in significant reduction of inflammation in the CNS associated with reduction in demyelination, axonal loss and ongoing damage (Wang et al., 2006). In the present study, reduction in the inflammatory cells in the spinal cords was very well reflected in the clinical scores of mice which dropped from 1.5 and stayed at $0.5-0$ in the RTL551treated group. Not only did the number of already infiltrated cells in the spinal cord diminish in response to RTL therapy, but the absence of cells in the cords both at the end of the $5 \mathrm{~d}$ treatment 
period and three days post treatment suggests that the treatment blocked entry of additional antigen specific cells into spinal cords. In contrast, the number of infiltrating green cells increased with time in the spinal cords of vehicle treated mice (Fig. 4).

The infiltrating cells were not undergoing apoptosis in the CNS, as demonstrated by TUNEL staining. Therefore, such a dramatic reduction of inflammatory infiltrates in the spinal cords of treated mice prompted us to study the spatial expression of various chemokines and receptors in the spinal cords. All the studied chemokines were down-regulated in the RTL-treated group, most notably CXCL2 and CCL6 as presented above. This is in accordance with the fact that only CX3CL1 (Fractalkine) and SDF1 $\alpha /$ CXCL12 are constitutively expressed in CNS by neurons and astrocytes respectively (Cartier et al., 2005) and, therefore, all the other chemokines appeared to be up-regulated as a part of the disease process. On the other hand, chemokine receptors are expressed on oligodendrocytes, astrocytes and neurons along with the inflammatory cells. In both chronic and acute MS lesions but not in healthy brains, immunoreactivity was demonstrated for MCP1/CCL2, MCP2/CCL8, CCL3, and CCL7 (McManus et al., 1998; Van Der Voorn et al., 1999). Among others, CCL5, CCL3 and CCL4 were detected in actively demyelinating MS plaques with high levels of demyelination. Among the receptors, CCR1, 2, $3, \& 5$ were detected on macrophages and activated microglia in chronic active lesions (Simpson et al., 2000). Moreover, CCR8 expressed on phagocytic macrophages and activated microglia has been correlated with ongoing demyelination in MS lesions (Trebst et al., 2003). Krumbholz et al., detected CXCL13 within the perivascular infiltrates in the active demyelinating lesions but not in chronic inactive lesions and normal CNS (Krumbholz et al., 2006). CXCL1, 8, \& 10 chemokines were also detected at high levels in the hypertrophic astrocytes within MS lesions (Omari et al., 2005). Taken together, these data show that chemokines and receptors are up-regulated in MS as well as EAE, and therefore, can serve as important targets for therapeutic intervention.

Various studies have demonstrated the role of chemokines and their receptors in the recruitment of monocytes and T-cells in the spinal cords of mice with EAE (Szczuciński and Losy, 2007). The down-regulation of chemokines and their respective receptors in the spinal cords of RTL551-treated mice might result in the loss of a chemotactic gradient leading to reduction in the migration of antigen specific cells in the spinal cord parenchyma. Besides chemokines and receptors, the interaction between beta1 and beta2 family integrins expressed on lymphocytes in conjunction with VCAM-1 and ICAM-1 expressed on endothelial cells drives the migration of lymphocytes through the blood brain barrier in EAE. Retention of inflammatory infiltrates in the perivascular space, as demonstrated in TNFR1 null mice, prevents the formation of demyelinating lesions in the spinal cord (Gimenez et al., 2004). Interestingly in the present study, both adhesion molecules, VCAM-1 and ICAM-1, were strongly downregulated on the spinal cord vascular endothelium in RTL551treated mice, providing further mechanistic support for the absence of inflammatory infiltrates in the spinal cords. Along with the endothelium, ICAM-1 is also expressed on leukocytes, neurons and glial cells (Bullard et al., 2007). Similarly, VCAM-1 expression on endothelium and astrocytes facilitates the entry and retention of inflammatory infiltrates into the parenchyma of spinal cords in EAE (Barten and Ruddle, 1994; Vajkoczy et al., 2001; Gimenez et al., 2004). TNF $\alpha$ has been shown to increase the up-regulation of VCAM-1 on astrocytes and endothelial cells. In accordance with this, TNF $\alpha$ mRNA was down-regulated eightfold in the spinal cords of the RTL551-treated mice.
In summary, our results demonstrate for the first time that effective treatment of EAE with RTLs involves a direct reduction of pro-inflammatory cytokines IL-17 and TNF $\alpha$ by encephalitogenic cells and vastly inhibited migration of inflammatory cells into the CNS mediated through a striking reduction in the expression of chemokines/receptors and VCAM-1 and ICAM-1 in affected spinal cord tissue.

\section{References}

Asensio VC, Lassmann S, Pagenstecher A, Steffensen SC, Henriksen SJ, Campbell IL (1999) C10 is a novel chemokine expressed in experimental inflammatory demyelinating disorders that promotes recruitment of macrophages to the central nervous system. Am J Pathol 154:1181-1191.

Baker D, Butler D, Scallon BJ, O’Neill JK, Turk JL, Feldmann M (1994) Control of established experimental allergic encephalomyelitis by inhibition of tumor necrosis factor (TNF) activity within the central nervous system using monoclonal antibodies and TNF receptor-immunoglobulin fusion proteins. Eur J Immunol 24:2040-2048.

Barten DM, Ruddle NH (1994) Vascular cell adhesion molecule-1 modulation by tumor necrosis factor in experimental allergic encephalomyelitis. J Neuroimmunol 51:123-133.

Bettelli E, Oukka M, Kuchroo VK (2007) T(H)-17 cells in the circle of immunity and autoimmunity. Nat Immunol 8:345-350.

Bitsch A, Kuhlmann T, Da Costa C, Bunkowski S, Polak T, Brück W (2000) Tumor necrosis factor alpha mRNA expression in early multiple sclerosis lesions: correlation with demyelinating activity and oligodendrocyte pathology. Glia 29:366-375.

Bullard DC, Hu X, Schoeb TR, Collins RG, Beaudet AL, Barnum SR (2007) Intercellular adhesion molecule-1 expression is required on multiple cell types for the development of experimental autoimmune encephalomyelitis. J Immunol 178:851-857.

Burrows GG, Bebo BF, Adlard KL, Vandenbark AA, Offner H (1998) Twodomain MHC class II molecules form stable complexes with myelin basic protein 69-89 peptide that detect and inhibit rat encephalitogenic T cells and treat experimental autoimmune encephalomyelitis. J Immunol 161:5987-5996.

Burrows GG, Chang JW, Bächinger HP, Bourdette DN, Offner H, Vandenbark AA (1999) Design, engineering and production of functional single-chain T cell receptor ligands. Protein Eng 12:771-778.

Burrows GG, Chou YK, Wang C, Chang JW, Finn TP, Culbertson NE, Kim J, Bourdette DN, Lewinsohn DA, Lewinsohn DM, Ikeda M, Yoshioka T, Allen CN, Offner H, Vandenbark AA (2001) Rudimentary TCR signaling triggers default IL-10 secretion by human Th1 cells. J Immunol 167:4386-4395.

Cartier L, Hartley O, Dubois-Dauphin M, Krause KH (2005) Chemokine receptors in the central nervous system: role in brain inflammation and neurodegenerative diseases. Brain Res Brain Res Rev 48:16-42.

Chang JW, Mechling DE, Bächinger HP, Burrows GG (2001) Design, engineering, and production of human recombinant $\mathrm{T}$ cell receptor ligands derived from human leukocyte antigen DR2. J Biol Chem 276:24170-24176.

Chaudhary P, Marracci GH, Bourdette DN (2006) Lipoic acid inhibits expression of ICAM-1 and VCAM-1 by CNS endothelial cells and T cell migration into the spinal cord in experimental autoimmune encephalomyelitis. J Neuroimmunol 175:87-96.

Chen Y, Langrish CL, McKenzie B, Joyce-Shaikh B, Stumhofer JS, McClanahan T, Blumenschein W, Churakovsa T, Low J, Presta L, Hunter CA, Kastelein RA, Cua DJ (2006) Anti-IL-23 therapy inhibits multiple inflammatory pathways and ameliorates autoimmune encephalomyelitis. J Clin Invest 116:1317-1326.

Eltayeb S, Berg AL, Lassmann H, Wallström E, Nilsson M, Olsson T, EricssonDahlstrand A, Sunnemark D (2007) Temporal expression and cellular origin of CC chemokine receptors CCR1, CCR2 and CCR5 in the central nervous system: insight into mechanisms of MOG-induced EAE. J Neuroinflammation 4:14

Fontenot AP, Keizer TS, McCleskey M, Mack DG, Meza-Romero R, Huan J, Edwards DM, Chou YK, Vandenbark AA, Scott B, Burrows GG (2006) Recombinant HLA-DP2 binds beryllium and tolerizes beryllium-specific pathogenic CD4+ T cells. J Immunol 177:3874-3883.

Gimenez MA, Sim JE, Russell JH (2004) TNFR1-dependent VCAM-1 expression by astrocytes exposes the CNS to destructive inflammation. J Neuroimmunol 151:116-125. 
Glabinski AR, Krakowski M, Han Y, Owens T, Ransohoff RM (1999) Chemokine expression in GKO mice (lacking interferon-gamma) with experimental autoimmune encephalomyelitis. J Neurovirol 5:95-101.

Glabinski AR, O’Bryant S, Selmaj K, Ransohoff RM (2000) CXC chemokine receptors expression during chronic relapsing experimental autoimmune encephalomyelitis. Ann N Y Acad Sci 917:135-144.

Haines JL, Ter-Minassian M, Bazyk A, Gusella JF, Kim DJ, Terwedow H, Pericak-Vance MA, Rimmler JB, Haynes CS, Roses AD, Lee A, Shaner B, Menold M, Seboun E, Fitoussi RP, Gartioux C, Reyes C, Ribierre F, Gyapay G, Weissenbach J, Hauser SL, Goodkin DE, Lincoln R, Usuku K, Oksenberg JR (1996) A complete genomic screen for multiple sclerosis underscores a role for the major histocompatability complex. The Multiple Sclerosis Genetics Group. Nat Genet 13:469-471.

Huan J, Subramanian S, Jones R, Rich C, Link J, Mooney J, Bourdette DN, Vandenbark AA, Burrows GG, Offner H (2004) Monomeric recombinant TCR ligand reduces relapse rate and severity of experimental autoimmune encephalomyelitis in SJL/J mice through cytokine switch. J Immunol 172:4556-4566.

Huo Y, Weber C, Forlow SB, Sperandio M, Thatte J, Mack M, Jung S, Littman DR, Ley K (2001) The chemokine KC, but not monocyte chemoattractant protein-1, triggers monocyte arrest on early atherosclerotic endothelium. J Clin Invest 108:1307-1314.

Izikson L, Klein RS, Charo IF, Weiner HL, Luster AD (2000) Resistance to experimental autoimmune encephalomyelitis in mice lacking the CC chemokine receptor (CCR)2. J Exp Med 192:1075-1080.

Kanno M, Suzuki S, Fujiwara T, Yokoyama A, Sakamoto A, Takahashi H, Imai Y, Tanaka J (2005) Functional expression of CCL6 by rat microglia: a possible role of CCL6 in cell-cell communication. J Neuroimmunol 167:72-80

Kerr BJ, Patterson PH (2004) Potent pro-inflammatory actions of leukemia inhibitory factor in the spinal cord of the adult mouse. Exp Neurol 188:391-407.

Komiyama Y, Nakae S, Matsuki T, Nambu A, Ishigame H, Kakuta S, Sudo K, Iwakura Y (2006) IL-17 plays an important role in the development of experimental autoimmune encephalomyelitis. J Immunol 177:566-573.

Krumbholz M, Theil D, Cepok S, Hemmer B, Kivisäkk P, Ransohoff RM, Hofbauer M, Farina C, Derfuss T, Hartle C, Newcombe J, Hohlfeld R, Meinl E (2006) Chemokines in multiple sclerosis: CXCL12 and CXCL13 up-regulation is differentially linked to CNS immune cell recruitment. Brain 129:200-211.

Langrish CL, Chen Y, Blumenschein WM, Mattson J, Basham B, Sedgwick JD, McClanahan T, Kastelein RA, Cua DJ (2005) IL-23 drives a pathogenic $\mathrm{T}$ cell population that induces autoimmune inflammation. J Exp Med 201:233-240.

Livak KJ, Schmittgen TD (2001) Analysis of relative gene expression data using real-time quantitative PCR and the 2(-Delta Delta C(T)) Method. Methods 25:402-408.

Luo Y, Fischer FR, Hancock WW, Dorf ME (2000) Macrophage inflammatory protein-2 and $\mathrm{KC}$ induce chemokine production by mouse astrocytes. J Immunol 165:4015-4023.

Mahad DJ, Ransohoff RM (2003) The role of MCP-1 (CCL2) and CCR2 in multiple sclerosis and experimental autoimmune encephalomyelitis (EAE). Semin Immunol 15:23-32.

Martino G, Consiglio A, Franciotta DM, Corti A, Filippi M, Vandenbroeck K, Sciacca FL, Comi G, Grimaldi LM (1997) Tumor necrosis factor alpha and its receptors in relapsing-remitting multiple sclerosis. J Neurol Sci 152:51-61.

Matejuk A, Dwyer J, Ito A, Bruender Z, Vandenbark AA, Offner H (2002) Effects of cytokine deficiency on chemokine expression in CNS of mice with EAE. J Neurosci Res 67:680-688.

McManus C, Berman JW, Brett FM, Staunton H, Farrell M, Brosnan CF (1998) MCP-1, MCP-2 and MCP-3 expression in multiple sclerosis le- sions: an immunohistochemical and in situ hybridization study. J Neuroimmunol 86:20-29.

Offner H, Subramanian S, Wang C, Afentoulis M, Vandenbark AA, Huan J, Burrows GG (2005) Treatment of passive experimental autoimmune encephalomyelitis in SJL mice with a recombinant TCR ligand induces IL-13 and prevents axonal injury. J Immunol 175:4103-4111.

Omari KM, John GR, Sealfon SC, Raine CS (2005) CXC chemokine receptors on human oligodendrocytes: implications for multiple sclerosis. Brain 128:1003-1015.

Park H, Li Z, Yang XO, Chang SH, Nurieva R, Wang YH, Wang Y, Hood L, Zhu Z, Tian Q, Dong C (2005) A distinct lineage of CD4 T cells regulates tissue inflammation by producing interleukin 17. Nat Immunol 6:1133-1141.

Rentzos M, Nikolaou C, Rombos A, Voumvourakis K, Segditsa I, Papageorgiou C (1996) Tumour necrosis factor alpha is elevated in serum and cerebrospinal fluid in multiple sclerosis and inflammatory neuropathies. J Neurol 243:165-170.

Rottman JB, Slavin AJ, Silva R, Weiner HL, Gerard CG, Hancock WW (2000) Leukocyte recruitment during onset of experimental allergic encephalomyelitis is CCR1 dependent. Eur J Immunol 30:2372-2377.

Ruddle NH, Bergman CM, McGrath KM, Lingenheld EG, Grunnet ML Padula SJ, Clark RB (1990) An antibody to lymphotoxin and tumor necrosis factor prevents transfer of experimental allergic encephalomyelitis. J Exp Med 172:1193-1200.

Selmaj K, Raine CS, Cross AH (1991a) Anti-tumor necrosis factor therapy abrogates autoimmune demyelination. Ann Neurol 30:694-700.

Selmaj K, Raine CS, Cannella B, Brosnan CF (1991b) Identification of lymphotoxin and tumor necrosis factor in multiple sclerosis lesions. J Clin Invest 87:949-954.

Simpson J, Rezaie P, Newcombe J, Cuzner ML, Male D, Woodroofe MN (2000) Expression of the beta-chemokine receptors CCR2, CCR3 and CCR5 in multiple sclerosis central nervous system tissue. J Neuroimmunol 108:192-200.

Szczuciński A, Losy J (2007) Chemokines and chemokine receptors in multiple sclerosis. Potential targets for new therapies. Acta Neurol Scand 115:137-146.

Trebst C, Staugaitis SM, Kivisäkk P, Mahad D, Cathcart MK, Tucky B, Wei T, Rani MR, Horuk R, Aldape KD, Pardo CA, Lucchinetti CF, Lassmann H, Ransohoff RM (2003) CC chemokine receptor 8 in the central nervous system is associated with phagocytic macrophages. Am J Pathol 162:427-438

Vajkoczy P, Laschinger M, Engelhardt B (2001) Alpha4-integrin-VCAM-1 binding mediates $\mathrm{G}$ protein-independent capture of encephalitogenic $\mathrm{T}$ cell blasts to CNS white matter microvessels. J Clin Invest 108:557-565.

Vandenbark AA, Rich C, Mooney J, Zamora A, Wang C, Huan J, Fugger L, Offner H, Jones R, Burrows GG (2003) Recombinant TCR ligand induces tolerance to myelin oligodendrocyte glycoprotein $35-55$ peptide and reverses clinical and histological signs of chronic experimental autoimmune encephalomyelitis in HLA-DR2 transgenic mice. J Immunol 171:127-133.

Van Der Voorn P, Tekstra J, Beelen RH, Tensen CP, Van Der Valk P, De Groot CJ (1999) Expression of MCP-1 by reactive astrocytes in demyelinating multiple sclerosis lesions. Am J Pathol 154:45-51.

Wang C, Mooney JL, Meza-Romero R, Chou YK, Huan J, Vandenbark AA, Offner H, Burrows GG (2003) Recombinant TCR ligand induces early TCR signaling and a unique pattern of downstream activation. J Immunol 171:1934-1940.

Wang C, Gold BG, Kaler LJ, Yu X, Afentoulis ME, Burrows GG, Vandenbark AA, Bourdette DN, Offner H (2006) Antigen-specific therapy promotes repair of myelin and axonal damage in established EAE. J Neurochem 98:1817-1827. 